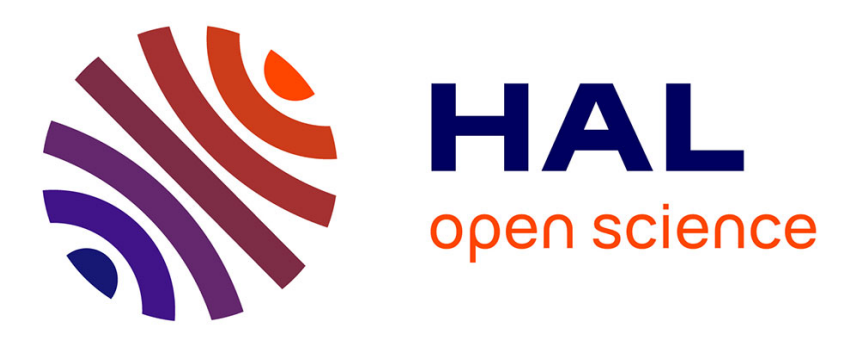

\title{
Stress-dependent moisture diffusion in composite materials
}

\author{
Georges Youssef, Sylvain Fréour, Frédéric Jacquemin
}

\section{To cite this version:}

Georges Youssef, Sylvain Fréour, Frédéric Jacquemin. Stress-dependent moisture diffusion in composite materials. Journal of Composite Materials, 2009, 43 (15), pp.1621-1637. 10.1177/0021998309339222 . hal-01007330

\section{HAL Id: hal-01007330 \\ https://hal.science/hal-01007330}

Submitted on 18 Nov 2017

HAL is a multi-disciplinary open access archive for the deposit and dissemination of scientific research documents, whether they are published or not. The documents may come from teaching and research institutions in France or abroad, or from public or private research centers.
L'archive ouverte pluridisciplinaire HAL, est destinée au dépôt et à la diffusion de documents scientifiques de niveau recherche, publiés ou non, émanant des établissements d'enseignement et de recherche français ou étrangers, des laboratoires publics ou privés. 


\title{
Stress-dependent Moisture Diffusion in Composite Materials
}

\author{
G. Youssef, S . F RE' OUR AND F. JACQUEMIN \\ Institut de Recherche en Génie Civil et Mécanique (UMR CNRS 6183) \\ Université de Nantes - Centrale Nantes, 37 Boulevard de l'Université \\ BP 406, 44602 Saint-Nazaire Cedex, France
}

\begin{abstract}
Experiments have indicated that the diffusion properties of a penetrant organic matrix composite system may change with time due to evolution of the internal mechanical strain states experienced by the constituting matrix of the composite plies. A multi-scale approach coupling the internal mechanical states, predicted by continuum medium mechanics, and their localization at the ply-constituent scale to the traditional Fick's law governing the moisture diffusion process was used in order to achieve the modeling of the response of composite laminates submitted to environmental hygroscopic loads, from the transient part of the diffusion process to its permanent stage. Various numerical practical cases were considered: the effects of the internal swelling strains on the time- and space-dependent diffusion coefficient, maximum moisture absorption capacity, moisture content, and states of internal stresses are extensively studied and discussed.
\end{abstract}

KEY WORDS: moisture diffusion, internal stresses, hygromechanical coupling, multi-scale modeling.

\section{INTRODUCTION}

$\mathbf{H}$ IGH PERFORMANCE COMPOSITES are being increasingly used in aerospace and marine structural applications. Organic matrix composites are often submitted to moisture and temperature environments. These environmental effects can lead to composite degradation and consequent loss of mechanical properties [1-4]. An essential feature of almost all combinations of weathering conditions is humidity; hence, topics pertinent to the question of performance in the presence of moisture are of prime importance. These topics are commonly divided into two subjects. The first relates to factors which drive moisture into the composites, namely, the penetration mechanisms. The second deals with the effects of the presence of water on the performance and durability of the composites. Several studies showed the important effects of humidity on the mechanical properties of composite materials and on their long-term behavior [5]; in particular, problems of chemical and 
physical aging and of dimensional stability (swelling) caused by internal stresses. Considerable efforts have been made by researchers to study the effects of moisture and temperature, and to develop analytical models for predicting the multi-scale mechanical states occurring during both the transient stage and the permanent regime of the moisture diffusion process of fiber-reinforced laminates submitted to hygro-mechanical loads [6-9]. In the previously cited references, the moisture diffusion process was assumed to follow the linear, classical, established for a long time, Fickian model. Nevertheless, some valuable experimental results, already reported [10], have shown that certain anomalies in the moisture sorption process, (i.e. discrepancies from the expected Fickian behavior) could be explained by basic principles of irreversible thermodynamics, by a strong coupling between the moisture transport in polymers and the local stress state $[11,12]$.

The effect of external loading on moisture penetration into a composite material is actually a markedly relevant issue since it is difficult to imagine any application of composite materials (even non-structural) which does not result in subjecting them to some form of static or dynamic loading. It has been claimed that the general effect of such loading is to enhance the moisture-penetration mechanisms producing higher rates and maximum levels of moisture penetration. As a result, aging mechanisms taking place under the effect of moisture are also enhanced, thus decreasing the durability of the material.

In the present study, a scale transition analysis is achieved in order to investigate the coupled effect of both the external and the internal mechanical states (stresses and strains) experienced by epoxy resin composite structures, on the moisture penetration process. This work is focused on the main penetration mechanism known in composite materials, namely, the diffusion into the bulk resin matrix. The model disregards the effect of external loading on damage-dependent mechanisms of capillary flow along the fiber-matrix interface and flow of moisture in micro-flaws occurring in the matrix. The hygro-mechanical model enables to account for the proper evolution of the water diffusion behavior parameters (rate of moisture absorption, diffusion coefficients, and maximum moisture content) of the epoxy resin constituting each ply of the composite structure, during the transient part of the diffusion process. The scale transition approach provides relations linking the ply stresses (strains) to those of the constituents (epoxy and reinforcements) and homogenization procedures enabling the estimation of the evolution of the plydiffusion behavior law, from that of the epoxy, at any step of the moisture diffusion. The present work underlines the effects induced by typical laminate lay-up configurations, whose diffusion behavior under stresses is compared to that of unidirectional composites. The numerical results, obtained according to the hygro-mechanical coupled model are also compared to those provided by the traditional uncoupled model.

\section{ACCOUNTING FOR A COUPLING BETWEEN THE MECHANICAL STATES AND THE MOISTURE DIFFUSION IN PURE ORGANIC MATRIX}

Polymer matrix constitutes the preferential penetration path for small molecule penetrants, such as water, diffusing through organic matrix composites, especially in the cases when their reinforcements are impermeable. Only dense polymer will be considered in the present work. Thus, according to this additional condition, the occurrence of voids or porosities will be neglected, in the following. Dense polymers have no pores, however, there exists the thermally agitated motion of chain segments providing penetrant-scale transient gaps (free volume) in the polymer matrix allowing penetrants to diffuse into 
the bulk of the material, from one side of the structure to the other [13]. The size and shape of the thermally induced cavities, available in polymers control the rate of gas diffusion and its permeation properties [14,15]. These cavities and packing irregularities actually constitute the so-called 'free-volume' of the material. The free-volume concept is extensively taken into account for explaining many properties presented by polymers, such as their visco-elastic behavior [16]. Especially, transport phenomena in polymers are generally explained by theories based on the extensive involvement of the free-volume notion [17]. The free volume actually corresponds to the difference between the specific (macroscopic) volume of the polymer and the actual volume occupied by its constitutive molecules. It is obvious, that the transport mobility of particles in a well-packed system depends on the degree of packing of the system, an inverse of measure of which is the matrix free-volume fraction: $v_{\mathrm{f}}^{\mathrm{m}}$, in the case that an organic matrix composite with impermeable reinforcements, is considered. Free-volume concept theoretical approaches, based on the works of Cohen and Turnbull [18] are often applied to the study of gas permeation. For instance, diffusion coefficients of gases in polymers can be deduced from the equations established by Fujita [19].

Moreover, it has also been realized, that the heterogeneous swelling, which accompanies sorption or desorption of water, leads to the creation of a multi-scale stress pattern in a composite structure. This aspect has been the subject of numerous papers over the past few years [2-4,20]. As early as 1953, Crank had suggested that the swelling stresses in polymer membranes, through which a penetrant diffuses, affects the diffusion coefficient [21]. The question is still addressed in the recent literature [22].

As a consequence, due to the heterogeneous moisture diffusion behavior of the epoxy resins, on the one hand, and that of the carbon fibers, on the other hand, the hygromechanical coupling occurring in composite structures initiates at the scale of the constituents, and especially within the organic matrix. In order to reach the goal of the present work, the modeling of the hygro-mechanical behavior of organic matrix composite laminates, a multi-scale is, thus, obviously required.

However, employing such a multi-scale approach implies as usual the knowledge of the specific behavior of the single elementary constituents of the representative elementary volume. As a consequence, the following paragraphs will be devoted to the study of the hygro-mechanical coupling existing in the single polymer matrix. According to the literature, this coupling affects both parameters governing the Fickean diffusion law:

(i) the diffusion coefficient, and

(ii) the maximum moisture absorption capacity.

\section{Moisture Diffusion Coefficient}

Following the experimental observations, a theoretical approach was suggested that was based on the calculation of the free-volume change in the stressed state. This corresponded with Fahmy and Hurt's [23] ideas; they calculated the free-volume change under stresses for an epoxy resin. This calculation is based on the modeling of the polymer by an assembly of thick spherical shells having the same ratio of inner to outer radii. The volume fraction of the spherical cavity, (being the same for all shells) represents the free-volume fraction of the organic matrix. In practice, it was later observed by Neumann and Marom [24,25], that the main mechanical state controlling the hygro-mechanical coupling was the strain instead of the stresses. Accordingly, the present work develop a model based on the strains. 
Assuming that the Fickean diffusion coefficient was related to the free volume by the Doolittle equation, the authors proposed an expression for the ratio of the diffusion coefficients in the strained and free-of-strain states [24,26]:

$$
\left.\ln \left(\frac{D_{\varepsilon}^{m}}{D_{0}^{m}}\right)=\frac{a}{v^{m}} \frac{1}{v_{f 0}^{m}}-\frac{1}{v_{f \varepsilon}^{m}}\right)
$$

where $D_{0}^{m}$ and $D_{\varepsilon}^{m}$ are the Fickean moisture diffusion coefficients for the strainfree matrix and that of the strained epoxy, respectively, whereas $a$ is an empirically deduced factor.

$v^{m}$ denotes the volume fraction of the matrix in the composite ply, $v_{f 0}^{m}$ and $v_{f \varepsilon}^{m}$ are the free-volume fractions of the strain-free epoxy and that of the strained organic matrix, respectively.

Many authors agree that the value of $v_{f 0}^{m}$ for a defect-free resin tends toward $2.5 \%[23,26]$.

The free-volume fraction for a strained epoxy is related to its counterpart existing in the corresponding unstrained resin through:

$$
v_{f \varepsilon}^{m}=v_{f 0}^{m}+\frac{\Delta V^{m}}{V_{0}^{m}}
$$

where $\Delta V^{m} / V_{0}^{m}$ stands for the organic matrix volume variation induced by the strains. It corresponds in practice to the trace of the strain tensor experienced by the polymer matrix $\operatorname{Tr} \varepsilon^{m}$ :

$$
\frac{\Delta V^{m}}{V_{0}^{m}}=\frac{V_{\varepsilon}^{m}-V_{0}^{m}}{V_{0}^{m}}=\operatorname{Tr} \varepsilon^{m}
$$

where $V_{\varepsilon}^{m}$ and $V_{0}^{m}$ denote the volume of the strain organic matrix and that of the unstrained epoxy, respectively.

Equations (1) and (2) yield the following expression for the Fickean moisture diffusion coefficients of the strained/unstrained resins:

$$
\ln \left(\frac{D_{\varepsilon}^{m}}{D_{0}^{m}}\right)=\frac{a}{v^{m}} \frac{\Delta V^{m} / V_{0}^{m}}{v_{f 0}^{m}\left[v_{f 0}^{m}+\Delta V^{m} / V_{0}^{m}\right]}
$$

In order to estimate the diffusion coefficient of the strained polymeric matrix $D_{\varepsilon}^{m}$, the knowledge of both the organic matrix strain tensor and its strain-free diffusion coefficient $D_{0}^{m}$ is required. $D_{0}^{m}$ can be deduced from the slope of the mass uptake evolution as a function of the time power $1 / 2$, when the time tends toward 0 (thus, at the beginning of the diffusion process). In the case that a N5208 epoxy is considered, the value of this coefficient, measured at room temperature is: $D_{0}^{m}=13.484 \times 10^{-8} \mathrm{~mm}^{2} \mathrm{~s}^{-1}$.

According to Equation (4), amorphous polymer matrices experiencing any kind of strain state are subjected to a variation of their free volume. Such a mechanical state controlled free-volume change necessarily involves an evolution of the water-like, small-molecule penetrant, diffusion law parameters. The moisture diffusion coefficient increases when the trace of the strain tensor experienced by the polymer is positive, and decreases when this trace is negative. This formalism is consistent with the practical observations. 
One observes actually in practice, that the diffusion coefficient of a pure epoxy matrix increases when it is submitted to an uniaxial tensile load, whereas this coefficient decreases when the polymer is submitted to an uniaxial compressive load [23].

The same authors state, in the same article, that the increased water uptake observed after submitting a polymer to tensile load is dependent not only on the diffusion coefficient but also on the equilibrium water concentration and that the stress state may indeed influence both of these parameters. According to Fahmy and Hurt [23] the hygro-mechanical coupling has not only an influence over the evolution of the diffusion coefficient, but on the maximum moisture absorption capacity, too. This second feature will be developed extensively in the next paragraph.

\section{Maximum Moisture Absorption Capacity}

The maximum moisture absorption capacity for an unstrained epoxy satisfies (see, for instance, [26]):

$$
M_{\infty 0}^{m}=v_{f 0}^{m} \times \frac{\rho^{w}}{\rho^{m}}
$$

Let us assume that the maximum moisture absorption capacity of the same organic matrix experiencing any strain state becomes:

$$
M_{\infty \varepsilon}^{m}=v_{f \varepsilon}^{m} \times \frac{\rho^{w}}{\rho^{m}}
$$

Equations (5) and (6) yield:

$$
M_{\infty \varepsilon}^{m}-M_{\infty 0}^{m}=\left(v_{f \varepsilon}^{m}-v_{f 0}^{m}\right) \times \frac{\rho^{w}}{\rho^{m}}
$$

Combining relations (2) and (7) leads to:

$$
M_{\infty \varepsilon}^{m}=M_{\infty 0}^{m}+\frac{\Delta V^{m}}{V_{0}^{m}} \times \frac{\rho^{w}}{\rho^{m}}
$$

According to the experimental results presented, for instance, by Neumann and Marom, [25], on pure epoxies, parameter $a$, appearing in Equation (3), especially, does vary in a narrow range: $0.031 \leq a \leq 0.036$ with an average value equal to 0.033 .

\section{COMPOSITE MATERIALS}

\section{Modeling the Moisture Diffusion Process}

Consider a laminated hollow cylinder, whose inner and outer radii are respectively $a$ and $b$, composed of $n$ plies delimitated by cylinders with radii $r_{i}$ and $r_{i+1}$.

To solve the Fick's equation, the boundary moisture contents have to be known. The moisture content at saturation is determined from the moisture content of the matrix as shown in Equation (8). The mixed law on the volumes reads:

$$
V^{I}=v^{f} \times V^{f}+v^{m} \times V^{m}
$$


where $V^{I}, V^{f}$, and $V^{m}$ are, respectively, the volumes of the composite, the fibers, and the matrix. $v^{f}$ and $v^{m}$ are, respectively, the volume fractions of the fibers and matrix. Equation (9) can be expressed by introducing the moisture contents:

$$
\rho^{I} \times M_{\infty \varepsilon}^{I}=v^{f} \times \rho^{f} \times M_{\infty \varepsilon}^{f}+v^{m} \times \rho^{m} \times M_{\infty \varepsilon}^{m}
$$

where $\rho^{I}, \rho^{f}$, and $\rho^{m}$ are, respectively, the densities of the composite, the fibers, and the matrix.

Since, the fibers do not absorb water, Equation (10) is simplified as follows:

$$
M_{\infty \varepsilon}^{I}=v^{m} \frac{\rho^{m}}{\rho^{I}} M_{\infty \varepsilon}^{m}
$$

The macroscopic moisture content (at ply scale) is a solution of the Fick's equation with a moisture diffusion coefficient dependent on the matrix one, which is dependent on the local mechanical state. The expression of the effective moisture diffusion coefficient as a function of the moisture diffusion coefficient of the matrix is [27].

$$
D_{\varepsilon}^{I}=D_{\varepsilon}^{m} \frac{1-v^{f}}{1+v^{f}}
$$

The moisture diffusion coefficient of the matrix is determined through Equation (3) from the local strains deduced from the localization of the macroscopic strains.

The hygro-mechanical coupling induces different moisture diffusion coefficients and moisture contents for the constitutive plies. Thus, the moisture flux is continuous at the interply and the moisture content is discontinuous [7].

The Fickian problem (13-14) can be expressed as:

$$
\begin{gathered}
\frac{\partial C_{i}}{\partial t}=D_{i}(t)\left[\frac{\partial^{2} C_{i}}{\partial r^{2}}+\frac{1}{r} \frac{\partial C_{i}}{\partial r}\right] \quad a<r<b, t>0, i=1 \text { to } n \\
\left\{\begin{aligned}
C_{i}\left(r_{i}, t\right) & =\alpha_{i+1} C_{i+1}\left(r_{i}, t\right) \\
D_{i}(t) \frac{\partial C_{i}\left(r_{i}, t\right)}{\partial r} & =D_{i+1}(t) \frac{\partial C_{i+1}\left(r_{i}, t\right)}{\partial r} \\
C(a, t) & =M_{\infty}^{a}(t) \text { and } C(b, t)=M_{\infty}^{b}(t) \\
C(r, 0) & =0
\end{aligned}\right.
\end{gathered}
$$

where $C_{i}(r, t)$ is the moisture content, $D_{i}(t)$ and $D_{i+1}(t)$ are the diffusion coefficients of two adjacent plies, $\alpha_{i+1}$ is a constant corresponding to the moisture content jump between two adjacent plies: $\alpha_{i+1}=\left(M_{\infty \varepsilon}^{I}\right)_{i+1} /\left(M_{\infty \varepsilon}^{I}\right)_{i}$; where $\left(M_{\infty \varepsilon}^{I}\right)_{i+1}$ and $\left(M_{\infty \varepsilon}^{I}\right)_{i}$ are the maximum moisture absorption capacity of the two adjacent plies deduced from that of the matrix. $M_{\infty}^{a}(t)$ and $M_{\infty}^{b}(t)$ are the boundary moisture contents.

This problem is solved by using the finite differences with an explicit scheme.

\section{Mechanical Modeling}

The macroscopic internal stresses are calculated by considering the homogenized properties and the classical equations of solid mechanics: constitutive laws of hygrovisco-elastic orthotropic materials, strain-displacement relationship, compatibility and equilibrium equations, and boundary conditions. The coupling between moisture diffusion 
and mechanical states involves an incremental resolution. The moisture-dependent behavior, between times $t_{i-1}$ and $t_{i}$, can be expressed as:

$$
\sigma_{i}^{I}-\sigma_{i-1}^{I}=\bar{L}^{I}:\left[\left(\varepsilon_{i}^{I}-\varepsilon_{i-1}^{I}\right)-\bar{\beta}^{I}\left(C_{i}^{I}-C_{i-1}^{I}\right)\right]
$$

where $\bar{L}^{I}$ is the average macroscopic stiffness between $t_{i-1}$ and $t_{i}, \bar{\beta}^{I}$ the corresponding hygroscopic expansion, $\varepsilon^{I}$ the hygroscopic strain, and $C$ the moisture content.

The corresponding local stresses in the constituents (fibers and matrix) are deduced by using Eshelby-Kröner hygro-mechanical scale transition model. By assuming that the elastic fibers do not absorb any moisture, the local stresses-strains relation in the reinforcements is:

$$
\sigma^{f}=L^{f}: \varepsilon^{f}
$$

Using the Eshelby's formalism leads to the following scale transition expression for the strains in the fibers:

$$
\varepsilon^{f}=\left(L^{f}+L^{I}: R^{I}\right)^{-1}:\left(\sigma^{I}+L^{I}: R^{I}: \varepsilon^{I}\right)
$$

Applying Equation (17), the macroscopic state (stresses and strains) and the homogenized properties can be predicted. If these conditions are satisfied, the local mechanical states in the epoxy matrix are provided by Hill's [28] strains and stresses average laws:

$$
\left\{\begin{array}{c}
\varepsilon^{m}=\frac{1}{v^{m}} \varepsilon^{I}-\frac{v^{f}}{v^{m}} \varepsilon^{f} \\
\sigma^{m}=\frac{1}{v^{m}} \sigma^{I}-\frac{v^{f}}{v^{m}} \sigma^{f}
\end{array}\right.
$$

Figure 1 shows the calculation scheme used to solve the hygro-mechanically coupled problem. At every step of the calculation, the moisture diffusion coefficients are estimated through the knowledge of the preceding step multi-scale internal mechanical state, and especially from the volume strain of the ply organic matrix.

\section{Numerical Results}

According to the above-described theoretical approach considered in order to account for the hygro-mechanical coupling regarding the moisture diffusion process, a timedependent evolution of the boundary conditions in terms of moisture content is expected to occur. Actually, this phenomenon results from the ply swelling induced by the hygroscopic expansion. As a consequence, the predicted numerical results should be closely dependent on the macroscopic maximum moisture absorption capacity $M_{0}^{I}$ of the strainfree macroscopic ply considered in order to initialize the iterative calculation process. Now, this very parameter cannot a priori be determined from direct measurements. Actually, at the beginning of the moisture diffusion process, only an infinitely thin layer from the composite structure does contain water. Moreover, in this specific case, the in-depth distribution of the moisture content presents the most important gradients (this effect will be clearly illustrated further in this section). For both these reasons, classical gravimetric measurements would not be easily achieved (because the mass variation would be weak in the explored specimen) and neither would it be representative of $M_{0}^{I}$ (because of the existing moisture content gradients). Nevertheless, in practice, the experimental results 
report that at the permanent state of the diffusion process, in a damage-free unidirectional composite containing $60 \%$ fiber volume fraction, which is not submitted to another load except being kept in air saturated with water (100\% relative humidity), the moisture content tends towards $1.5 \%$. An iterative calculation method was dedicated to the finding of the initial maximum moisture absorption capacity $M_{0}^{I}$ enabling to satisfy the condition, observed in practice $M_{\infty}^{I}=1.5 \%$ in the permanent state, when the hygroscopic expansion attains its peak. Through this iterative method, the initial maximum moisture absorption capacity of an unidirectional ply initially dry and strain-free, with $v^{f}=60 \%$ was identified to be as low as $M_{0}^{I}=0.73$. This parameter obviously is valid in when the hygro-mechanically coupled model is considered. In the case of the traditional uncoupled model being considered, since the maximum moisture absorption capacity is independent of the

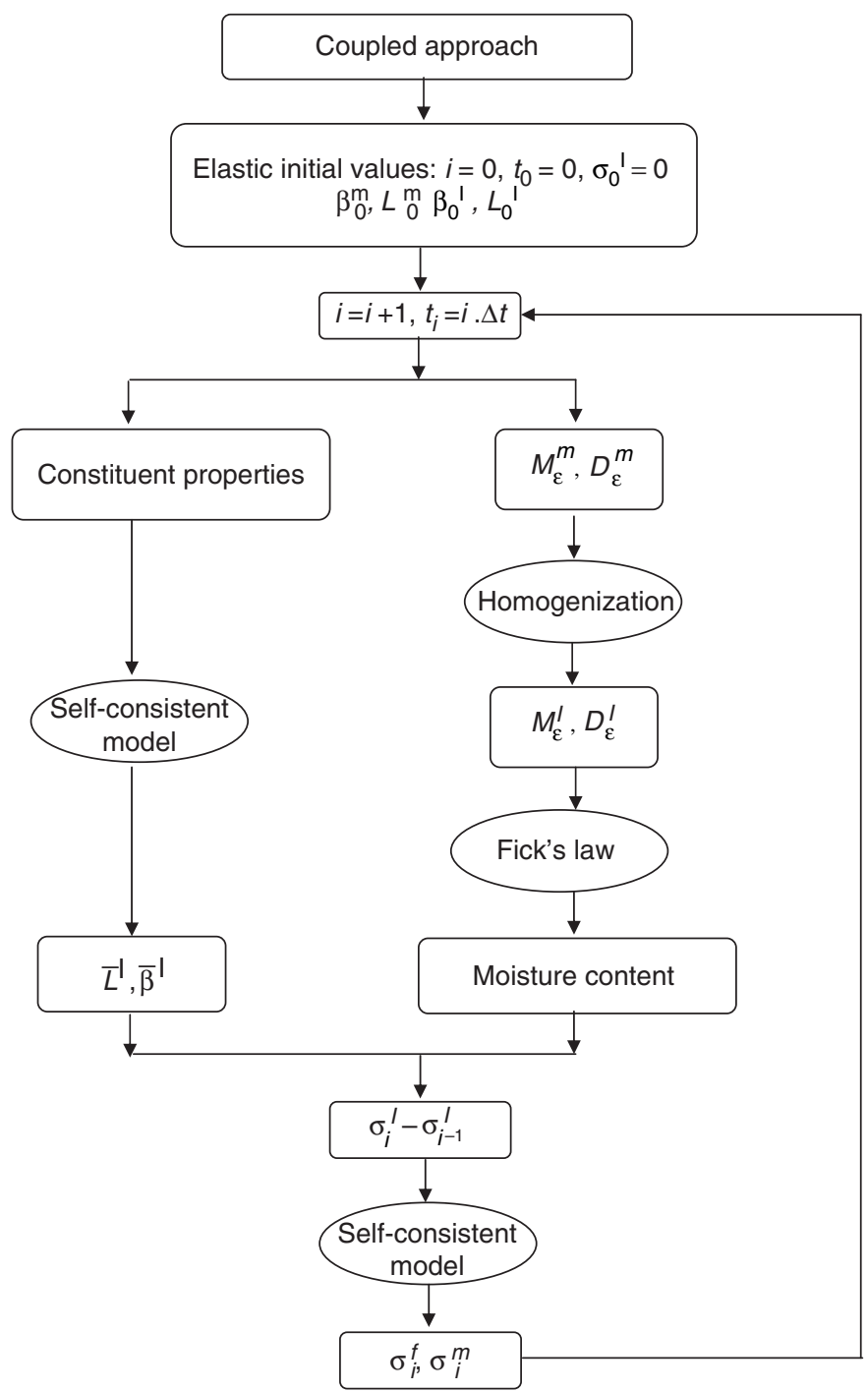

Figure 1. Calculation scheme. 
mechanical state, it does not vary throughout the diffusion process. As a consequence, the aimed final value $M_{\infty}^{I}=1.5 \%$ corresponds to the initial value, $M_{0}^{I}=1.5 \%$, as well.

The in-depth spatial and time-dependent macroscopic maximum moisture content profiles obtained in the case of $4 \mathrm{~mm}$ thick thin tubular composites structures submitted to a purely hygroscopic load are presented in Figure 2 below. Two geometrical arrangements of the composite plies were considered: (i) the first one (Figure 2(a)) corresponds to a $[+55 /-55]$ laminate, whereas (ii) the second one (Figure 2(b)) corresponds to a unidirectionally reinforced epoxy. Two sets of curves are displayed in Figure 2: the results obtained according to the coupled hygro-mechanical model are compared to those predicted by the traditional uncoupled model. In any case, in order to clearly take apart the effects generated by the hygro-mechanical coupling, the hygro-mechanical properties (elastic stiffness and coefficients of moisture expansion) of the very constituents of the composite ply (especially those of the organic matrix) were considered independent of the moisture content.

According to Figure 2, the coupled hygro-mechanical model satisfies the condition that the maximum moisture absorption capacity reaches the threshold $M_{\infty}^{I}=1.5 \%$ in the unidirectional structure (Figure 2(b)). In the case when the \pm 55 laminate is considered, the permanent state is attained for a maximum moisture absorption capacity limited to $M_{\infty}^{I}=1.37 \%$ only. This is actually due to a discrepancy between the mechanical strain states experienced by the two considered structures: the absolute value of $\operatorname{Tr} \varepsilon^{I}$ is weaker in the laminate than in the unidirectional composite. This affirmation clearly illustrates the contribution made by the coupled hygro-mechanical model, in comparison to the more

$\left(M_{\sigma}^{\circ}\right)^{l}(\%)$

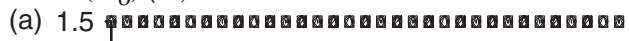

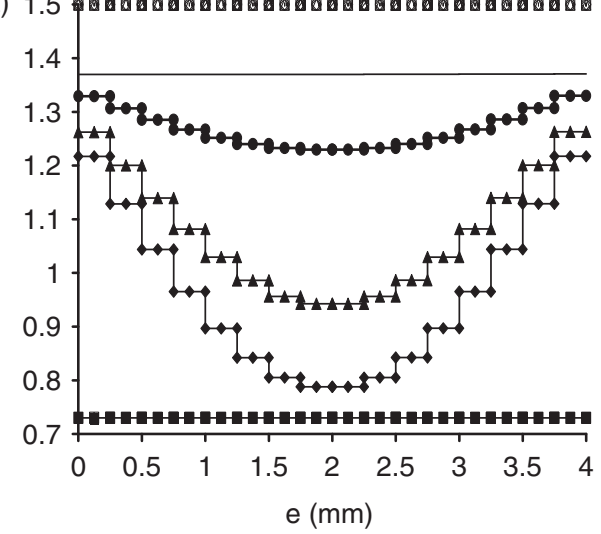

$\left(M_{\sigma}^{\infty}\right)^{l}(\%)$

(b) $1.5+00+000+0$

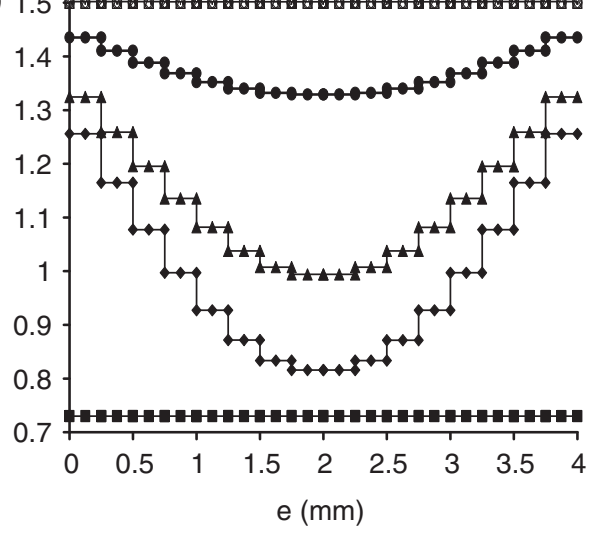

Coupled model Uncoupled model

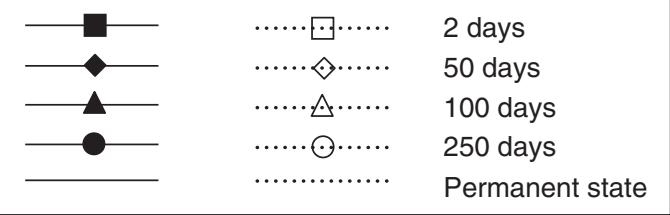

Figure 2. Comparison between the time- and space-dependent macroscopic permanent moisture absorption capacity profiles predicted by the coupled and uncoupled hygro-mechanical model in the (a) $\pm 55^{\circ}$ composite, (b) unidirectional composite structure. 
classical uncoupled approach. The uncoupled model is not capable of accounting for any change in the maximum absorption moisture capacity as a function of the time and space, during the transient part of the moisture diffusion process, neither does it have the capability to account for an evolution of this parameter as a function of the very arrangement of the composite plies in a laminated structure.

The present study underlines that the choice of the geometrical stacking of the composite plies, since it enables to optimize the multi-scale mechanical states experienced by the composite structure, should also enable significant optimization of the parameters of the moisture diffusion process, such as the maximum moisture absorption capacity or the moisture diffusion coefficient, so that the time- and depth-dependent moisture content profiles would also be significantly altered. One can expect that such substantial information would have an extensive effect on the durability predicted for composite structures designed for withstanding environmental loads. These results, foretold by the theoretical model obviously require a practical checking.

Moreover, it is noticeable in Figure 2, that contrary to the uncoupled model, the coupled hygro-mechanical model predicts stair-step-shaped discontinuities of the in-depth maximum moisture absorption capacity evolutions. The discontinuities are actually located at the boundaries between the laminate layers, which are considered to experience a homogeneous local mechanical state at the scale of the ply constituents. They come from this very distribution of the multi-scale mechanical states, and especially from that of the organic matrix, because the hygro-mechanical coupling initiates at the microscopic scale. This last assertion is compatible with the absence of discontinuities occurring at the beginning of the moisture diffusion process and when the permanent state is reached, both these situations corresponding to particular cases when the matrix strain is identical in each ply of the tubular structure. Nevertheless, the predictions regarding the initial and final steps of the diffusion process are specific to the considered problem. Actually, nonuniform distributions of the maximum moisture absorption capacity suggest themselves, in either of the cases wherein:

(i) the mechanical states are induced during the fabrication process,

(ii) or a thick tubular structure submitted to an external mechanical load.

Analogous effects do occur for the time- and space-dependent evolution of the macroscopic moisture diffusion coefficient, displayed in Figure 3 below:

Contrary to the previously discussed case of the maximum moisture absorption capacity, the moisture diffusion coefficient predicted by the coupled hygro-mechanical model does not depend much on the ply stratification, the relative deviation between Figure 3(a) and (b) remaining weaker than $10 \%$.

Figure 4 displays the various in-depth time-dependent evolutions of the moisture content in the studied hollow thin cylinders, calculated through both the coupled/uncoupled hygro-mechanical approaches.

According to Figure 4, the coupled hygro-mechanical model predicts a strong evolution of the moisture content at the boundaries of the tube (within the structure itself, obviously), during the whole diffusion process, even though the applied external purely hygroscopic load is kept constant. This phenomenon, that does not occur when the simulation is achieved according to the traditional uncoupled model, should be attributed to the variation of the maximum moisture absorption capacity, within the coupled framework, previously reported in Figure 2. 

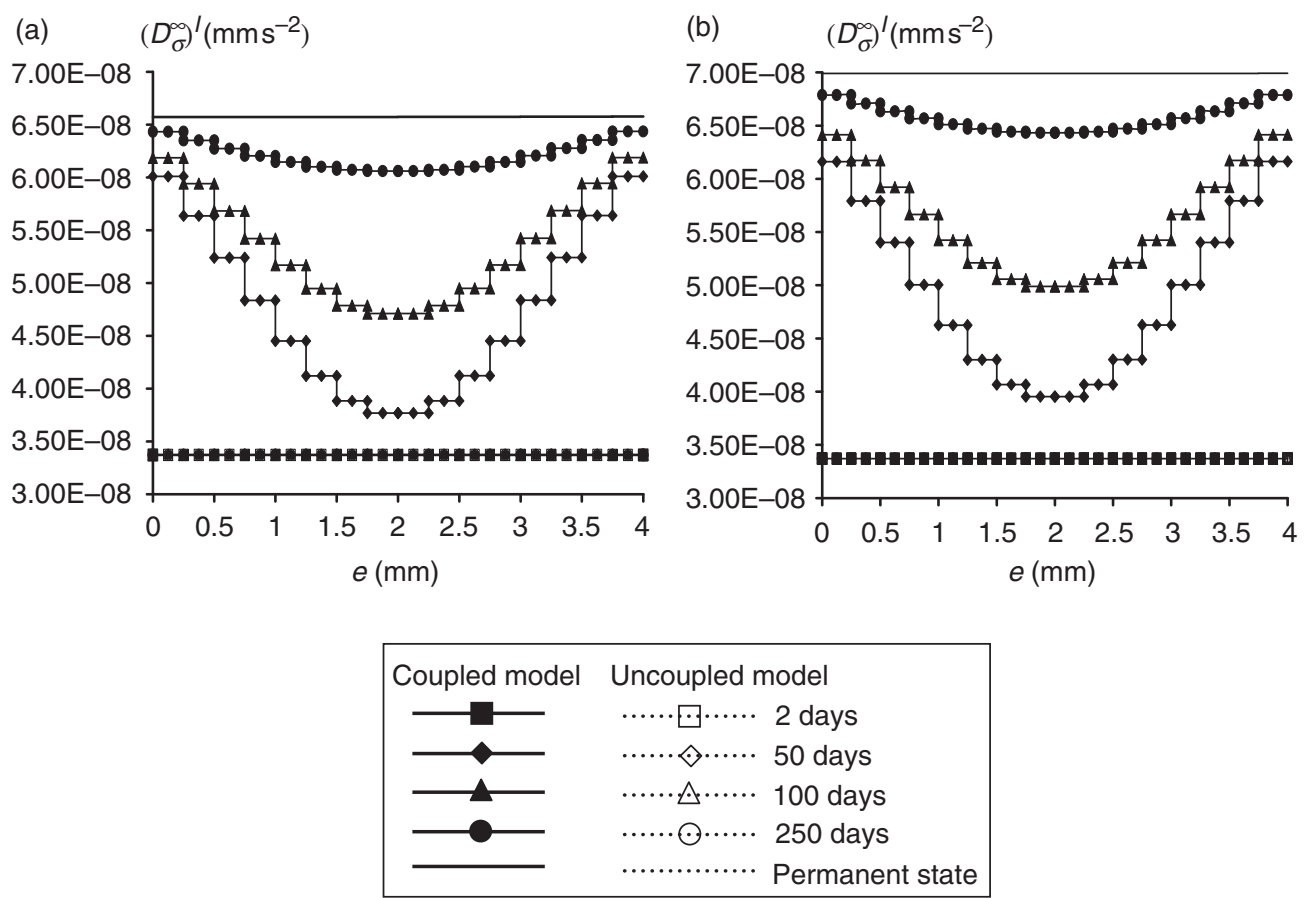

Figure 3. Comparison between the time- and space-dependent macroscopic moisture diffusion coefficient profiles predicted by the coupled and uncoupled hygro-mechanical model in the (a) $\pm 55^{\circ}$ composite, (b) unidirectional composite structure.

C (\%)

(a) 1.5

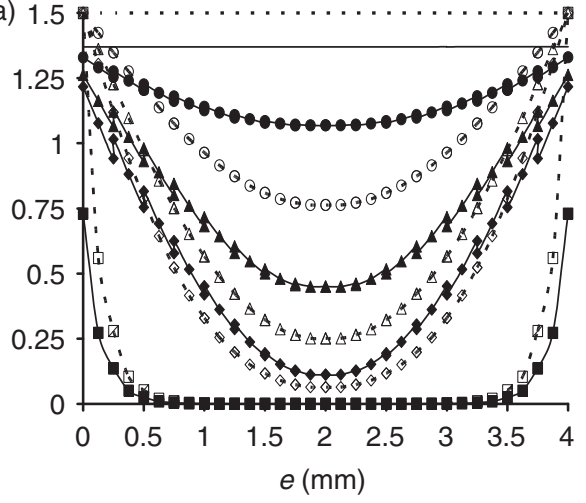

$C(\%)$

(b) 1.5

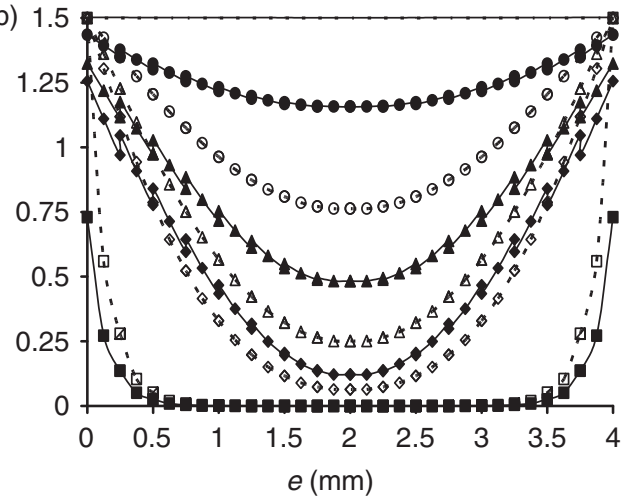

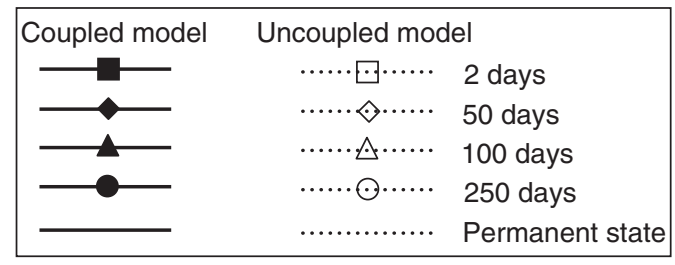

Figure 4. Time- and space-dependent moisture content profiles in the (a) $\pm 55^{\circ}$ composite structure, (b) unidirectional composite structure of coupled/un-coupled model. 
The considerable discrepancies observed between the moisture content profiles predicted by the coupled/uncoupled models are likely to result in a significant deviation of the multiscale internal stresses distribution too. This issue is extensively investigated in the next subsection.

\section{Predicted Multi-scale Mechanical States}

The following section of the present work is logically devoted to the analysis of the discrepancies occurring between the multi-scale mechanical stress evolutions predicted by i) the coupled hygro-mechanical model on the one hand, and ii) the uncoupled, one on the other.

Figure 5 describes the through-time multi-scale transverse stress state evolution in the internal ply of a) the laminated and b) the unidirectional cylinders, during the transient part of the diffusion process.

The coupled hygro-mechanical model predicted transverse stresses state the absolute value which is weaker than that predicted by the corresponding uncoupled approach. This result can be attributed to the fact that, as reported in Figure 4, the moisture content calculated through the coupled model is always weaker or identical to that deduced from the uncoupled ply. Whatever the considered geometrical arrangement of the plies, the most significant discrepancies between the transverse stresses predicted by the coupled/ uncoupled models occur at the beginning of the diffusion process. In the organic matrix, the absolute deviation can be as strong as $20 \mathrm{MPa}$ in a unidirectional structure. However, the discrepancy rapidly fades and is cancelled when the permanent state is attained, in this very structure (the moisture content is then identical whatever the considered calculation scheme (uncoupled/coupled), due to the constitutive assumptions

(a)

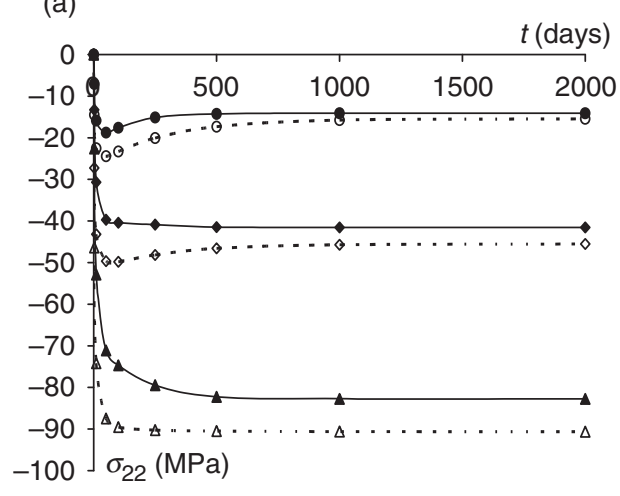

(b)

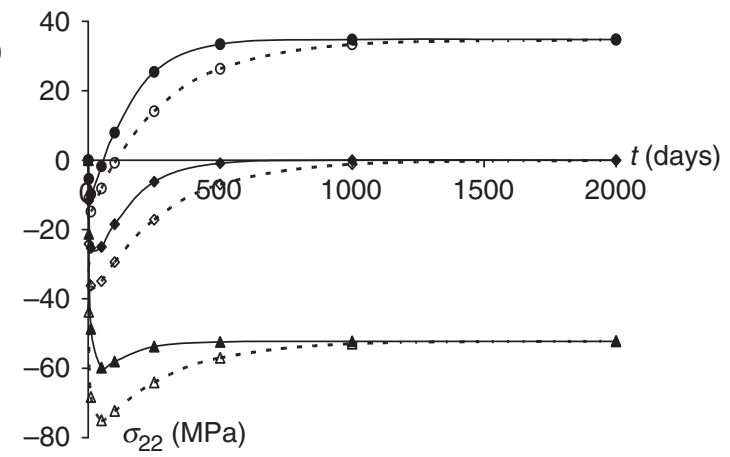

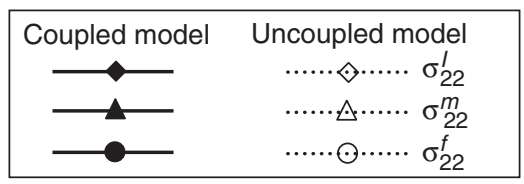

Figure 5. Multi-scale transversal stress states in the internal ply of the (a) $\pm 55^{\circ}$ laminate, (b) unidirectional composite structure. 
considered in the present work). On the other hand, in the laminate, an absolute deviation of $10 \mathrm{MPa}$ still holds in the permanent state of the diffusion process, regarding the predicted transverse stress experienced by the organic matrix.

In any case, the strongest relative deviation occurring between the transverse stresses calculated through the coupled/uncoupled models corresponds to the specific transient stage when the multi-scale stresses reach their extreme value, which is a deciding factor in the context of predicting the structure durability.

Figure 6 shows the comparison between the predicted in-plane shear stresses calculated in the internal ply of the laminated hollow cylinder.

It appears clearly in Figure 6 that during a rather short period of time, at the beginning of the diffusion process (namely, between 50 and 500 days), the shear stresses predicted according to the coupled approach increase faster, in absolute value, than those deduced from the uncoupled model. This effect has been attributed to the strongest local moisture content gradients induced by the discontinuities (see Figure 4) in the case of the coupled model being used in comparison with the uncoupled model, for this specific period of time. In the period following the diffusion process, the absolute values of the stresses predicted by the coupled approach fall under the levels reached according to the uncoupled model. The behavior is then similar to that described in the comments related to Figure 5.

A similar analysis was achieved in the central ply of the composite tube. Figure 7 shows the results obtained regarding the calculated multi-scale transverse stresses, whereas Figure 8 shows the evolution of the shear stresses.
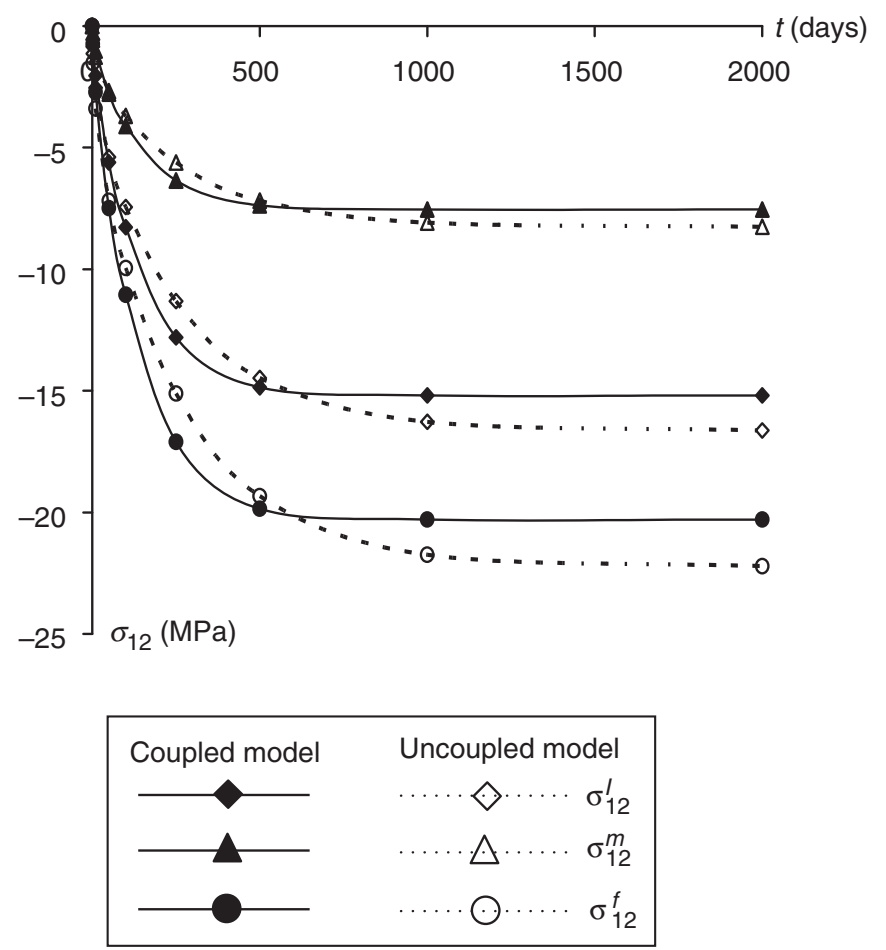

Figure 6. Multi-scale shear stress states in the internal ply of the $\pm 55^{\circ}$ composite laminate. 
(a)
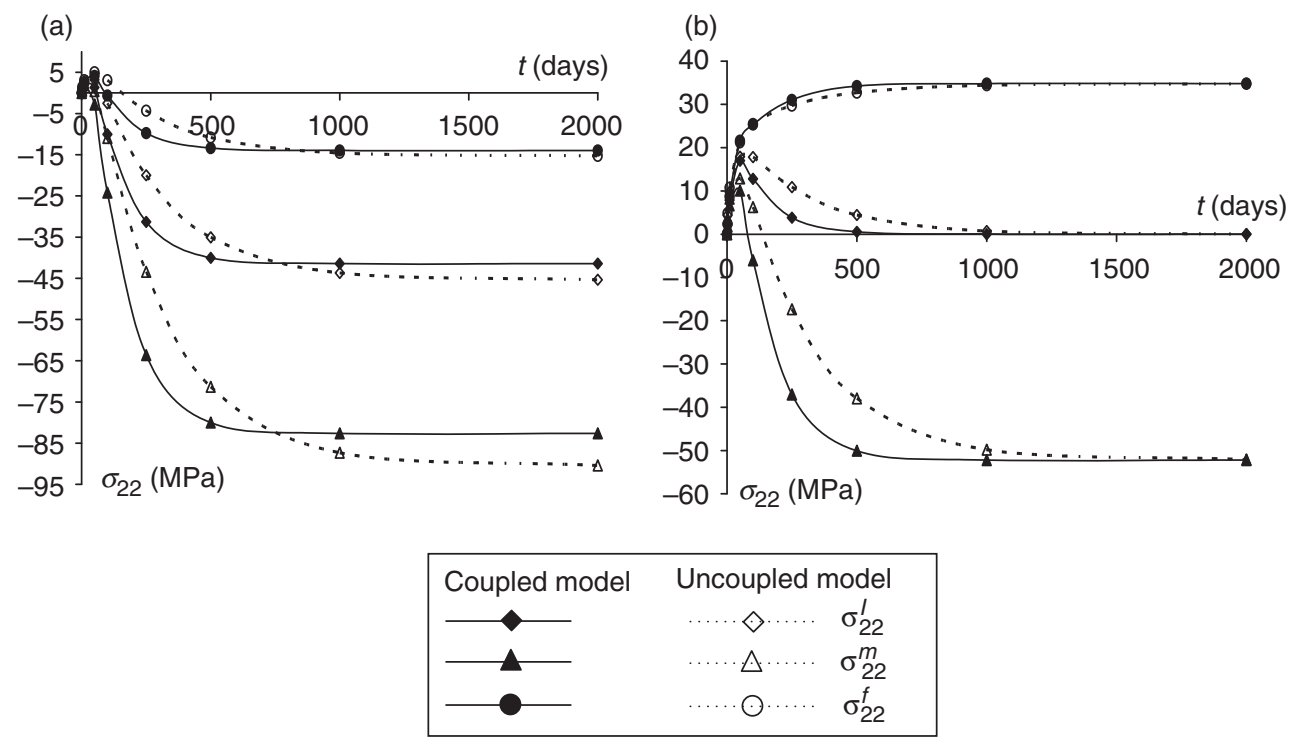

Figure 7. Multi-scale transverse stress states in the central ply of the (a) $\pm 55^{\circ}$ laminate, (b) unidirectional composite structure.

\section{Conclusion and Perspectives}

A multi-scale approach accounting for the existence of a hygro-mechanical coupling was used in the present work in order to determinethe time- and space-dependent internal stresses resulting from the purely hygroscopic loading of thin composite laminates. The coupling involves considering an evolution of the moisture transport process parameters as a function of the internal mechanical states, especially the volume strain of the organic matrix. The effective diffusion coefficient of the composite material is estimated from the homogenization procedure established by Hashin, accounting for the mechanical statedependent moisture diffusion coefficient of the constitutive epoxy. The hygro-mechanical coupling is assumed, as reported in the literature, to affect the maximum moisture absorption capacity. The in-depth moisture content evolution during the transient stage of the moisture diffusion process is deduced from Fick's law.

The macroscopic internal stresses are calculated from the classical continuum mechanics relationships whereas those of the plies' very constituents (organic matrix and its reinforcements, respectively) are deduced from Eshelby-Kröner analytical self-consistent model.

Accounting for the occurrence of the hygro-mechanical coupling yields a significant evolution of the moisture diffusion law governing parameters: the diffusion coefficient, and more noticeably the maximum moisture absorption capacity, each of them varying through the depth of the composite structure. The present study underlines that the choice of the geometrical stacking of the composite plies, since it enables to optimize the multiscale mechanical states experienced by the composite structure, also enables to significantly optimize the parameters of the moisture diffusion process, so that the time- and depth-dependent moisture content profiles would be significantly weaker than the corresponding profile predicted in an unidirectional structure. Moreover, the hygro-mechanical coupled model estimates that the multi-scale mechanical stresses are weaker than that 


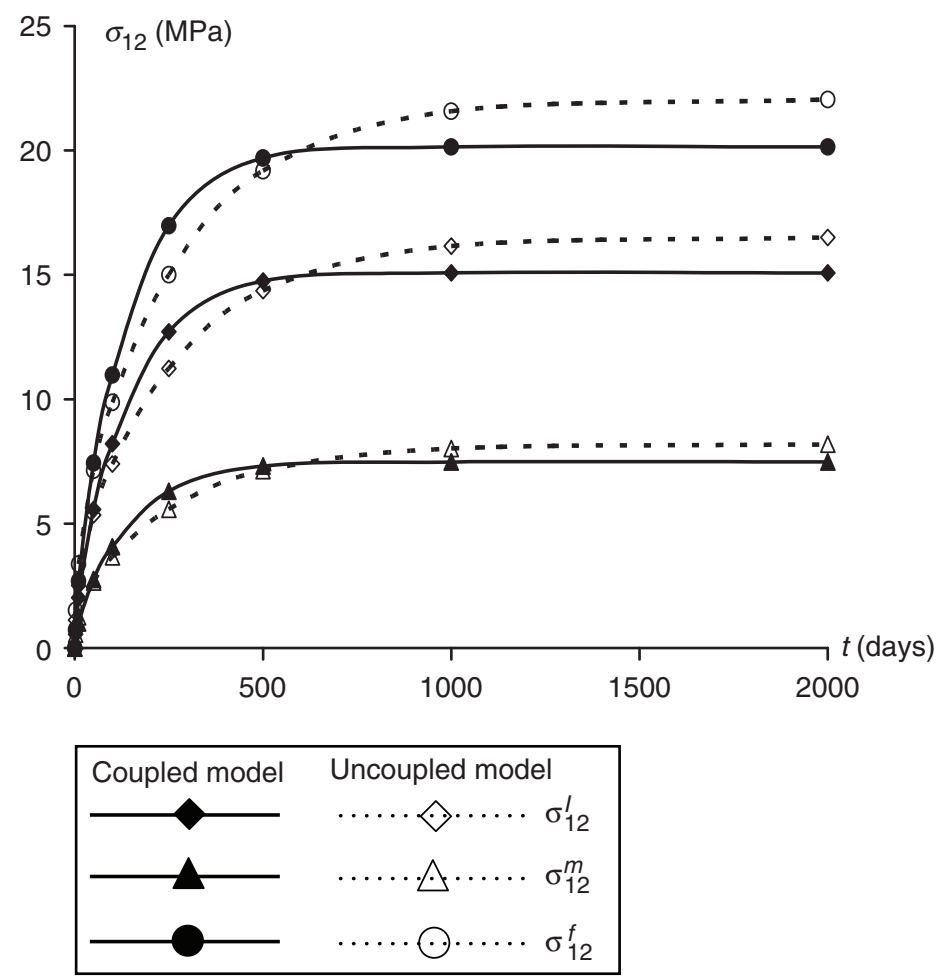

Figure 8. Multi-scale shear stress states in the central ply of the $\pm 55^{\circ}$ composite structure.

predicted by the traditional uncoupled model. Thus, the existence of the organic matrix volume strain effects on the moisture diffusion process should be considered as a significant parameter with regard to the sizing of composite structures conceived for withstanding hygro-mechanical loads during their service-life.

In further works, the evolution, reported in practice [29], of the organic matrix mechanical stiffness, as a function of its moisture content, will be considered in the multi-scale coupled approach presented in this work. Since according to Youssef et al. [30], to be published, this yields to the occurrence of an evolution of in-depth macroscopic properties during the transient part of the diffusion process, a dedicated, non-iterative homogenization scale transition procedure based on Mori-Tanaka estimates [31] instead of the presently used Eshelby-Kröner model [32], would be required.

Other published experiments reported that the measured diffusivities of carbon epoxy composites with long histories of exposure to sea water or to distilled water are higher by $35 \%-62 \%$ than the expected values. It was claimed that the considerable increase of the diffusivity had caused microdamage in the composites, creating more channels of water penetration [33]. Moreover, fiber debonding (consequence of material damage) enhances moisture penetration by capillary flow along the interface, according to the observations achieved by Field and Ashbee [34]. As a consequence, a scale transition model accounting for damage occurring in such fiber-epoxy composites will be developed in further works, so that it would be possible to introduce damage-related effects on the moisture diffusion behavior of the material. 


\section{REFERENCES}

1. Abou Msallem, Y., Boyard, N., Jacquemin, F., Poitou, A., Delaunay, D. and Chatel, S. (2008). Identification of Thermal and Rheological Properties of an Aeronautic Epoxy Resin-simulation Of Residual Stresses, International Journal of Material Forming, 1: 579-584.

2. Davies, P., Mazeas, F. and Casari, P. (2001). Sea Water Aging of Glass Reinforced Composites, Journal of Composite Materials, 35: 1343-1372.

3. Jedidi, J., Jacquemin, F. and Vautrin, A. (2005). Design of Accelerated Hygrothermal Cycles on Polymer Matrix Composites in the Case of a Supersonic Aircraft, Journal of Composite Structures, 68: 429-437.

4. Jedidi, J., Jacquemin, F. and Vautrin, A. (2006). Accelerated Hygrothermal Cyclical Tests for Carbon/Epoxy Laminates, Composites Part A: Applied Science and Manufacturing, 37: 636-645.

5. Shen, C.H. and Springer, G.S. (1977). Environmental Effects in the Elastic Moduli of Composite Materials, Journal of Composite Materials, 11: 250-264.

6. Gopalan, R., Rao, R.M.V.G.K., Murthy, M.V.V. and Dattaguru, B. (1986). Diffusion Studies on Advanced Fibre Hybrid Composites, Journal of Reinforced Plastics and Composites, 5: 51-61.

7. Jacquemin, F. and Vautrin, A. (2002). A Closed-form Solution for the Internal Stresses in Thick Composite Cylinders Induced by Cyclical Environmental Conditions, Composite Structures, 58: 1-9.

8. Fréour, S., Jacquemin, F. and Guillén, R. (2005). On an Analytical Self-consistent Model for Internal Stress Prediction in Fiber-reinforced Composites Submitted to Hygroelastic Load, Journal of Reinforced Plastics and Composites, 24: 1365-1377.

9. Jacquemin, F., Fréour, S. and Guillén, R. (2006). Analytical Modeling of Transient HygroElastic Stress Concentration - Application to Embedded Optical Fiber in a Non-uniform Transient Strain Field, Composites Science and Technology, 66: 397-406.

10. Gillat, O. and Broutman, L.J. (1978). Effect of External Stress on Moisture Diffusion and Degradation in a Graphite Reinforced Epoxy Laminate, ASTM STP, 658: 61-83.

11. Weitsman, Y. (1990a). A Continuum Diffusion Model for Viscoelastic Materials, Journal of Physical Chemistry, 94: 961-968.

12. Weitsman, Y. (1990b). Moisture in Composites: Sorption and Damage, In: Reifsnider, K.L. (ed.), Fatigue of Composite Materials, pp. 385-429, Elsevier Science Publisher, Amsterdam.

13. Chen, C., Han, B., Li, J., Shag, T., Zou, J. and Jiang, W. (2001). A New Model on the Diffusion of Small Molecule Penetrants in Dense Polymer Membranes, Journal of Membrane Science, 187: $109-119$.

14. Adamson, M.J. (1980). Thermal Expansion and Swelling of Cured Epoxy Resin Used in Graphite/Epoxy Composite Materials, Journal of Materials Science, 15: 1736-1745.

15. Wang, Z.F., Wang, B., Yang, Y.R. and Hu, C.P. (2003). Correlations Between Gas Permeation and Free-Volume Hole Properties of Polyurethane Membranes, European Polymer Journal, 39: 2345-2349.

16. Vaughan, D.J. and McPherson, E.L. (1973). The Effects of Adverse Environmental Conditions on the Resin-Glass Interface of Epoxy Composites, Composites, 4: 131-133.

17. Crank, J. and Park, G.S. (1968). Diffusion in Polymers, Academic Press Inc, London.

18. Cohen, M.H. and Turnbull, D. (1959). Molecular Transport in Liquids and Glasses, Journal of Chemical Physics, 31(5): 1164-1169.

19. Fujita, H. (1991). Notes on Free Volume Theories, Polymer Journal, 23(12): 1499-1506.

20. Gigliotti, M., Jacquemin, F., Molimard, J. and Vautrin, A. (2007). Transient and Cyclical Hygrothermoelastic Stress in Laminated Composite Plates: Modelling and Experimental Assessment, Mechanics of Materials, 39: 729-745.

21. Crank, J. (1953). A Theoretical Investigation of the Influence of Molecular Relaxation and Internal Stress on Diffusion in Polymers, Journal of Polymer Science, 11: 151-168. 
22. Larobina, D., Lavorgna, M., Mensitieri, G., Musto, P. and Vautrin, A. (2007). Water Diffusion in Glassy Polymers and their Silica Hybrids: An Analysis of State of Water Molecules and of the Effect of Tensile Stress, Macromolecular Symposia, 247: 11-20.

23. Fahmy, A.A. and Hurt, J.C. (1980). Stress Dependence of Water Diffusion in Epoxy Resin, Polymer Composites, 1: 77-80.

24. Neumann, S. and Marom, G. (1985). Stress Dependence of the Coefficient of Moisture Diffusion in Composite Materials, Polymer Composites, 6: 9-12.

25. Neumann, S. and Marom, G. (1987). Prediction of Moisture Diffusion Parameters in Composite Materials Under Stress, Journal of Composite Materials, 21: 68-80.

26. Neumann, S. and Marom, G. (1986). Free-volume Dependent Moisture Diffusion Under Stress in Composite Materials, Journal of Materials Science, 21: 26-30.

27. Hashin, Z. (1972). Theory of Fibre Reinforced Materials, NASA CR-1974.

28. Hill, R. (1967). The Essential Structure of Constitutive Laws for Metals Composites and Polycrystals, Journal of the Mechanics and Physics of Solids, 15: 79-95.

29. Sai Ram, K.S. and Sinha, P.K. (1991). Hygrothermal Effects on the Bending Characteristics of Laminated Composite Plates, Computational Structure, 40(4): 1009-1015.

30. Youssef, G., Fréour, S. and Jacquemin, F. Effects of Moisture Dependent Constituents Properties on the Hygroscopic Stresses Experienced by Composite Structures, Mechanics of Composite Materials (to be published).

31. Fréour, S., Jacquemin, F. and Guillén, R. (2006). Extension of Mori-Tanaka Approach to Hygroelastic Loading of Fiber-reinforced Composites - Comparison with Eshelby-Kröner Self-consistent Model, Journal of Reinforced Plastics and Composites, 25: 1039-1053.

32. Jacquemin, F., Fréour, S. and Guillén, R. (2005). A Hygroelastic Self-consistent Model for Fiber-reinforced Composites, Journal of Reinforced Plastics and Composites, 24: 485-502.

33. Mazor, A., Broutman, L.J. and Eckstein, B.H. (1978). Effect of Long-term Water Exposure on Properties of Carbon and Graphite Fiber Reinforced Epoxies, Polymer Engineering Science, 18(5): 341-349.

34. Field, S.Y. and Ashbee, K.H.G. (1972). Weathering of Fibre Reinforced Plastics. Progress of Debonding Detected in Model Systems by Using Fibres as Light Pipes, Polymer Engineering Science, 12: 30-33. 